\title{
Primary pontine haemorrhage: clinical and computed tomographic correlations
}

\author{
LEON A WEISBERG
}

From the Department of Neurology and Psychiatry of the Tulane Medical Center, the Department of Neurology of the Charity Hospital of New Orleans, and the Department of Neurology of the Veterans Administration Hospital of New Orleans, USA

SUMMARY The clinical and computerised tomographic findings in $\mathbf{4 0}$ patients with primary pontine haemorrhage were reviewed. Twenty-nine patients were hypertensive. Four patients had angiographic or necropsy evidence of vascular malformations. In 33 cases, there was rapid deterioration to maximal neurological deficit; whereas in seven cases, there was sudden onset but subsequent progression to maximal deficit 24 hours to 5 days following the initial ictus. Seven patients had clinical features considered atypical for pontine haemorrhage. Five patients survived and four of these were capable of performing activities of daily living within 3 months of the haemorrhage. In all cases CT showed a hyperdense non-enhancing brain stem haematoma. There was evidence of ventricular extension in 27 cases. There was CT evidence of subarachnoid blood in only two patients who also had vascular malformations. In 26 cases, there was CT evidence that the haematoma extended to the midbrain and in four cases to the thalamic region. In six cases $\mathrm{CT}$ was repeated 6 to 21 days after the initial scan and it showed resolution of the haematoma in size and density; none of the haematomas showed post-contrast enhancement on initial or follow-up CT.

Primary pontine haemorrhage is frequently caused by hypertensive cerebrovascular disease. The haemorrhage develops from degenerative arteriolar wall changes (lipohyalinosis, fibrinoid necrosis, microaneurysms). These patients usually present with characteristic neurological findings, which include sudden onset of coma, quadriplegia, miotic but light reactive pupils, respiratory disturbances, impaired horizontal eye movements (including absent caloric response). There is usually rapid neurological deterioration and death frequently occurs within $\mathbf{4 8}$ hours of the initial ictus. $^{1-5}$

Since the introduction of CT, it has become possible to delineate certain less common clinical findings and a somewhat different clinical course in some patients with primary pontine haemorrhage. This has prompted us to review 40 cases of the disorder to determine the clinical spectrum of this condition based upon CT findings.

Address for reprint requests: Leon A Weisberg MD, Department of Neurology and Psychiatry, 1430 Tulane Avenue, New Orleans, LA 70112, USA.

Received 24 May 1985 and in revised form 20 July 1985. Accepted 3 August 1985

\section{Methods and clinical material}

Forty patients with primary pontine haemorrhage diagnosed by CT were analysed. These patients were admitted to the neurology service of the Charity Hospital of New Orleans, Veterans Hospital of New Orleans, or Tulane University Medical Center Hospital. The clinical and CT findings were reviewed by the author. All patients were scanned with a Picker Synerview high-resolution body scanner (section thickness, $10 \mathrm{~mm}$; matrix size, $256 \times 256$; scan time one second) or General Electric high-resolution CT/T 8800 body scanner (section thickness, $10 \mathrm{~mm}$; matrix size, $256 \times 256$; scan time, 4.8 or 9.6 seconds).

\section{Clinical findings}

There were 28 men and 12 women. Patient age ranged from 28 to 69 years old ( 2 were in the twenties; 3 were in the thirties; 20 were in the forties; 11 were in the fifties; 4 were in the sixties). Twenty-four of the 29 hypertensive patients had chest radiographic or electrocardiographic evidence of cardiac enlargement, funduscopic evidence of hypertensive retinal change or clinical findings consistent with congestive heart failure. Three patients in the sixties were normotensive and four other younger patients were also normotensive. These patients had no other predisposing conditions for the haemorrhage; however amyloid angiopathy is excluded only by necropsy findings and this was unfortunately not done in 

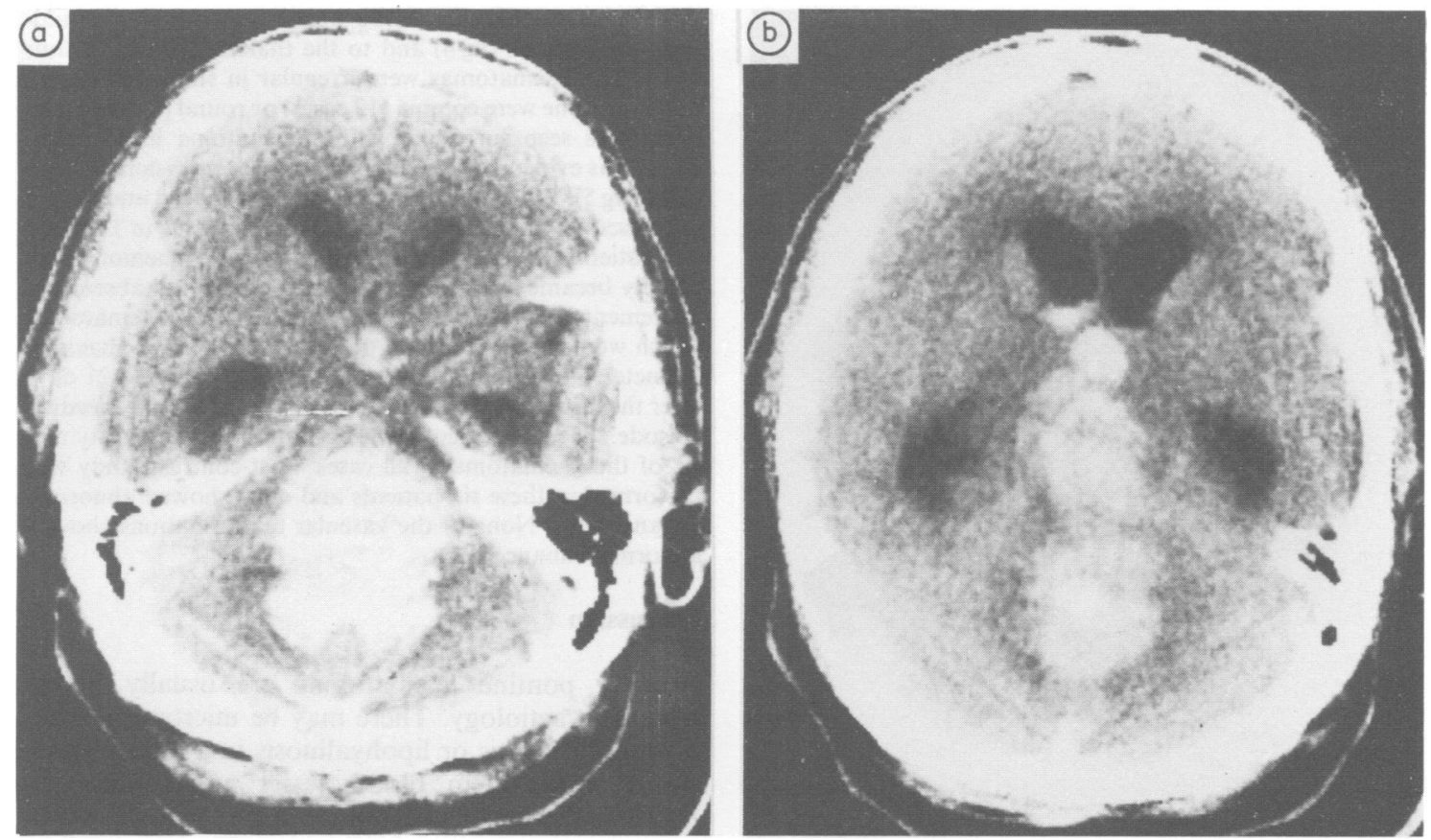

Fig 1 Fifty-year-old hypertensive man developed severe headache and then collapsed. He was comatose with irregular respiration, miotic pupils, absent caloric response and quadriplegia. CT findings: massive primary pontine haemorrhage (a) with midbrain and intraventricular extension (b).

these cases. Four patients had an underlying vascular malformation.

The onset of neurological disturbances was sudden in all patients. Ten patients reported severe headache immediately before neurological signs developed (fig 1). Episodes of transient neurological disturbances preceding the clinical ictus were not seen in any patient. The neurological findings in these patients are listed in table 1 . In 28 patients there was rapid progression to a comatose condition (fig 2 ). These patients also showed respiratory abnormalities, absent oculocephalic and caloric responses, quadriplegia with bilateral Babinski signs. Twenty-two of these patients had miotic but light reactive pupils, whereas six had fixed and dilated pupils. These patients with fixed and dilated pupils had the largest brain stem haemorrhages with ventricular extension. Five patients were initially lethargic and rapidly progressed to an obtunded state within 6 hours. These patients had respiratory abnormalities, quadriplegia, miotic but light reactive pupils. Three had absents horizontal eye movements including absent cabric response and two had conjugate gaze palsy.

Seven patients had sudden onset of neurological signs; however, there was progression of neurological signs for 48 hours to 5 days after the initial onset (fig 3 ). These patients were initially alert and two progressively had deterioration in the level of consciousness. These patients had hemiplegia. Four had miotic but light reactive pupils and three patients had unequal pupils. Four patients had conjugate gaze and three had internuclear ophthalmoplegia.
Thirty-one patients died within one week of the initial clinical episode. Four patients survived for 8 to 50 days but without showing significant neurological recovery. They died of secondary complications. Five patients survived the primary pontine haemorrhage. Four of these were capable of ambulating independently and were able to care for them-

Table 1 Initial neurological findings in 40 patients with primary pontine haemorrhage

\begin{tabular}{lr}
\hline Neurological signs & Number of cases \\
\hline Altered consciousness & 5 \\
lethargy-obtundation & 28 \\
stupor-coma & 25 \\
Respiratory abnormalities & 6 \\
irregular pattern & 2 \\
neurogenic hyperventilation & \\
Cheyne-Stokes pattern & 33 \\
Motor dysfunction & 7 \\
quadriplegia & \\
hemiplegia & 31 \\
Pupillary abnormalities & 6 \\
miotic and reactive & 3 \\
dilated and fixed & \\
unequal & 31 \\
Ocular motility disturbance & 6 \\
absent horizontal movements & 3 \\
conjugate gaze palsy & \\
internuclear ophthalmoplegia & 4 \\
Other neurological disturbances & 2 \\
cerebellar ataxia & \\
sensory deficit &
\end{tabular}




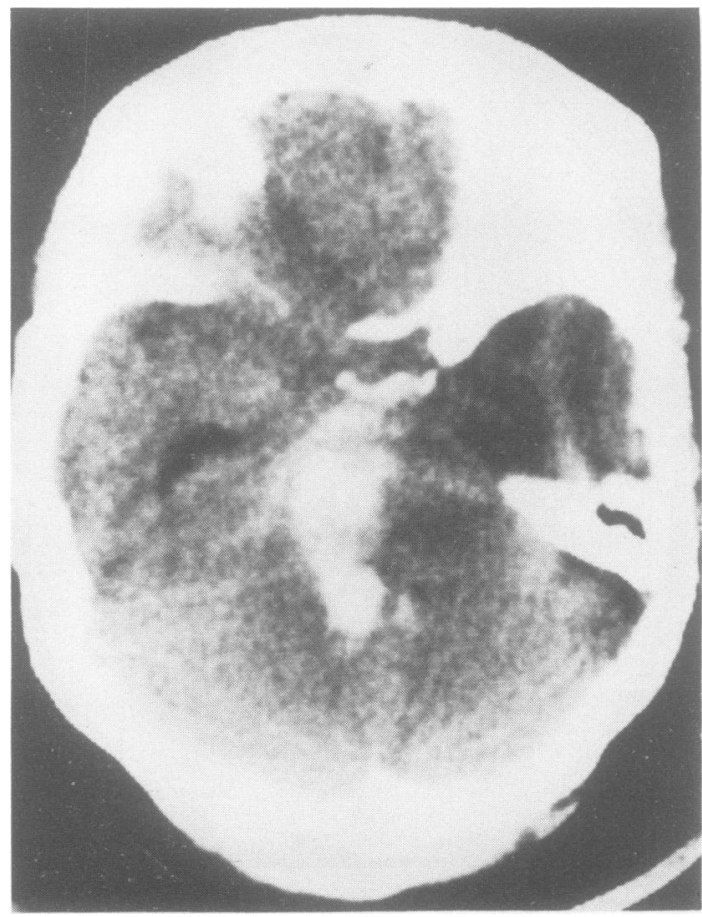

Fig 2 Forty-eight-year-old hypertensive woman suddenly collapsed. Findings were coma, hyperventilation, quadriplegia, miotic pupils and absent caloric response. CT findings: eccentric comma-shaped primary pontine haematoma involving tegmentum and basis pontis.

selves. One survivor did not recover sufficiently to ambulate independently and he required assistance with activities of daily living.

The CT findings in patients with primary pontine haemorrhage are listed in table 2 . Thirty-three of the haematomas were bilateral, involving tegmentum and basis pontis. The blood extended into the fourth ventricle in 27

Table 2 CT findings in 40 patients with primary pontine haemorrhage

\begin{tabular}{lc}
\hline CT findings & Number of cases \\
\hline Haematoma shape & \\
irregular & 19 \\
semilunar or comma & 12 \\
$\quad$ round & 9 \\
Location & 33 \\
$\quad$ medial & 7 \\
$\quad$ lateral & 27 \\
Ventricular extension of blood & 2 \\
Blood seen in the basal cisterns & 35 \\
Effacement of brain stem cisterns & \\
Extension of the haematoma & 26 \\
$\quad$ midbrain & 4 \\
thalamus & 11 \\
Edema & 17 \\
Hydrocephalus & \\
\hline
\end{tabular}

cases. Haemorrhage could be traced upwards to the midbrain in 26 cases (fig 4) and to the thalamic region in four cases. The haematomas were irregular in shape (19 cases); however some were comma (12 cases) or round ( 9 cases). Oedema was seen surrounding the haematoma in 11 cases. There was evidence of blood in the brain stem cisterns in two cases (fig 5). The brain stem cisterns were effaced and poorly visualised in 35 cases. There was hydrocephalus in 17 cases. All patients with tegmental-basilar pontine haemorrhages rapidly became comatose with quadriplegia and absent eye movements. There were eight patients with haematomas which were either unilateral or less than $18 \mathrm{~mm}$ in maximal diameter. Follow-up CT was performed within 6 to 21 days after the initial scan in six patients who survived the bleeding episode. There was significant resolution of the density and six of the haematoma in all cases. Post-contrast study was performed in these six patients and none showed abnormal enhancement. None of the vascular malformations showed abnormal enhancement.

\section{Discussion}

Primary pontine haematomas are usually hypertensive in aetiology. There may be microaneurysms, fibrinoid necrosis or lipohyalinosis in the deep penetrating brain stem blood vessel walls. The haemorrhage usually originates from vessels which measure 100 to $200 \mu \mathrm{m}$ in diameter. The source of the brain stem haemorrhage may include the paramedian penetrating branches of the basilar artery or lateral penetrating branches originating from the short circumferential branches of the basilar artery. Depending upon the specific location and size of the vessel from which the haemorrhage has originated, there may be a wider spectrum of clinical abnormalities than had been initially described by necropsy studies of patients with primary pontine haemorrhage.$^{1-3}$ If there is bleeding from a larger calibre arteriole, the haemorrhage is likely to be medial, involve both basis pontis and tegmentum, and extends to the midbrain. Bleeding from a smaller calibre arteriole which has a lateral location is more likely to cause a smaller haemorrhage. The lateral tegmental haematomas are usually smaller, associated with less severe neurological deficit, and less commonly fatal than the larger medial tegmental-basis pontis haematomas. ${ }^{6-11}$ In these 40 patients, the largest primary pontine haemorrhage occurred in those patients who where hypertensive and the smaller haematomas were seen in normotensive patients.

Several CT studies have reported certain less commonly recognised features of primary pontine haemorrhage. ${ }^{6-11}$ In seven of our cases, the onset was abrupt but this was followed by neurological deterioration which occurred 1-5 days later. In these cases, repeat CT did not show enlargement of haematoma, increased mass effect oedema or secondary development of intraventricular extension. In patients with 

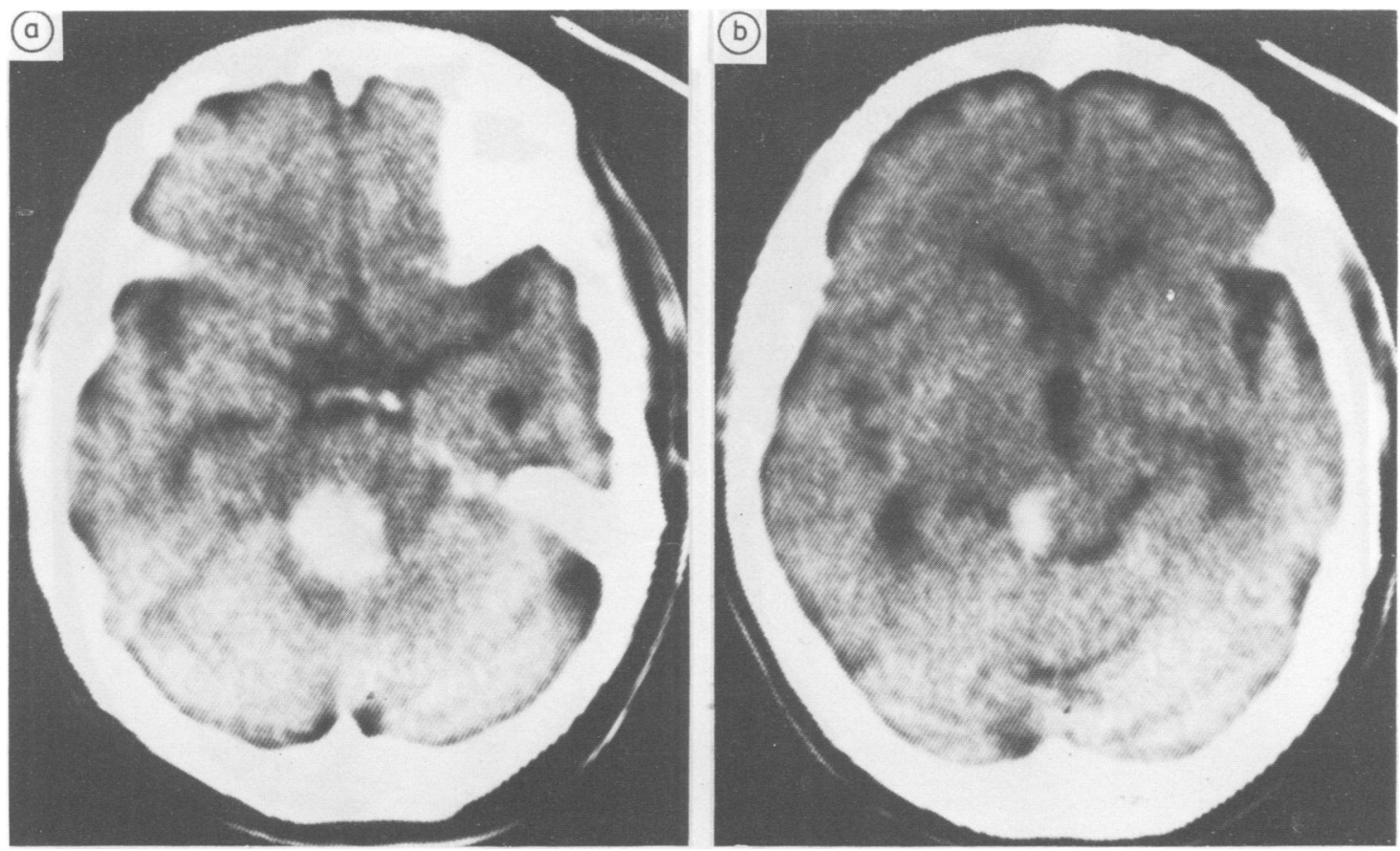

Fig 3 Sixty-nine-year-old normotensive man experienced headache and developed left hemiparesis and right facial droop. He was alert and had a right gaze paresis. CT findings: right (readers left) tegmental haematoma (a). There is extension to the lateral portion of the midbrain $(b)$. Four days later he neurologically deteriorated to an obtunded condition with quadriplegia. Follow-up CT (not shown) showed no change in the size or density of the haematoma.

smaller and lateral tegmental haematomas, several partial syndromes have been reported. These include the following: (1) ipsilateral gaze palsy and internuclear ophthalmoplegia, (2) small asymmetric pupils with the smaller pupil ipsilateral to the haematoma, (3) limb ataxia, (4) contralateral isolated hemiparesis, (5) contralateral hemianaesthesia. Seven of our patients were initially alert, seven patients had hemiplegia, six had conjugate gaze palsy, and three had internuclear ophthalmoplegia. In patients with these findings, brain stem infarct due to branch occlusion of the basilar artery was initially considered more likely than primary pontine haemorrhage.

Almost all patients with primary pontine haemorrhage have a fatal outcome. There have been several reports of prolonged survival in patients with documented CT evidence of primary pontine haemorrhage who recovered spontaneously. ${ }^{6-9}$ In addition, there have been reports of patients who recovered following surgical evacuation of the haematoma. ${ }^{1213}$ None of our patients underwent surgical evacuation of the brain stem haemorrhage. Five of the 40 patients in this series survived the primary pontine haemorrhage and four were capable of per- forming activities of daily living within 3 months. All patients who survived the primary pontine haemorrhage had no evidence of systemic arterial hypertension and only one had angiographic evidence of brain stem vascular malformation. The finding that outcome was better in normotensive than in hypertensive patients is consistent with the findings of another study of intracranial haemorrhage. ${ }^{14}$ All patients who survived had unilateral and more laterally located tegmental haemorrhages and none showed intraventricular extension.

Four patients with primary pontine haemorrhage had brain stem vascular malformations. These patients were normotensive. In two cases angiography showed evidence of an arteriovenous malformation; whereas in two cases angiography was negative but necropsy findings showed brain stem telangiectasia. In these four cases CT showed a hyperdense haematoma but post-contrast scan did not show evidence of abnormal enhancement. Based upon the CT characteristics there were no features to distinguish the aetiologies of the primary pontine haemorrhage; however in two cases due to vascular malformations blood was seen in the cisternal spaces. The presence of 

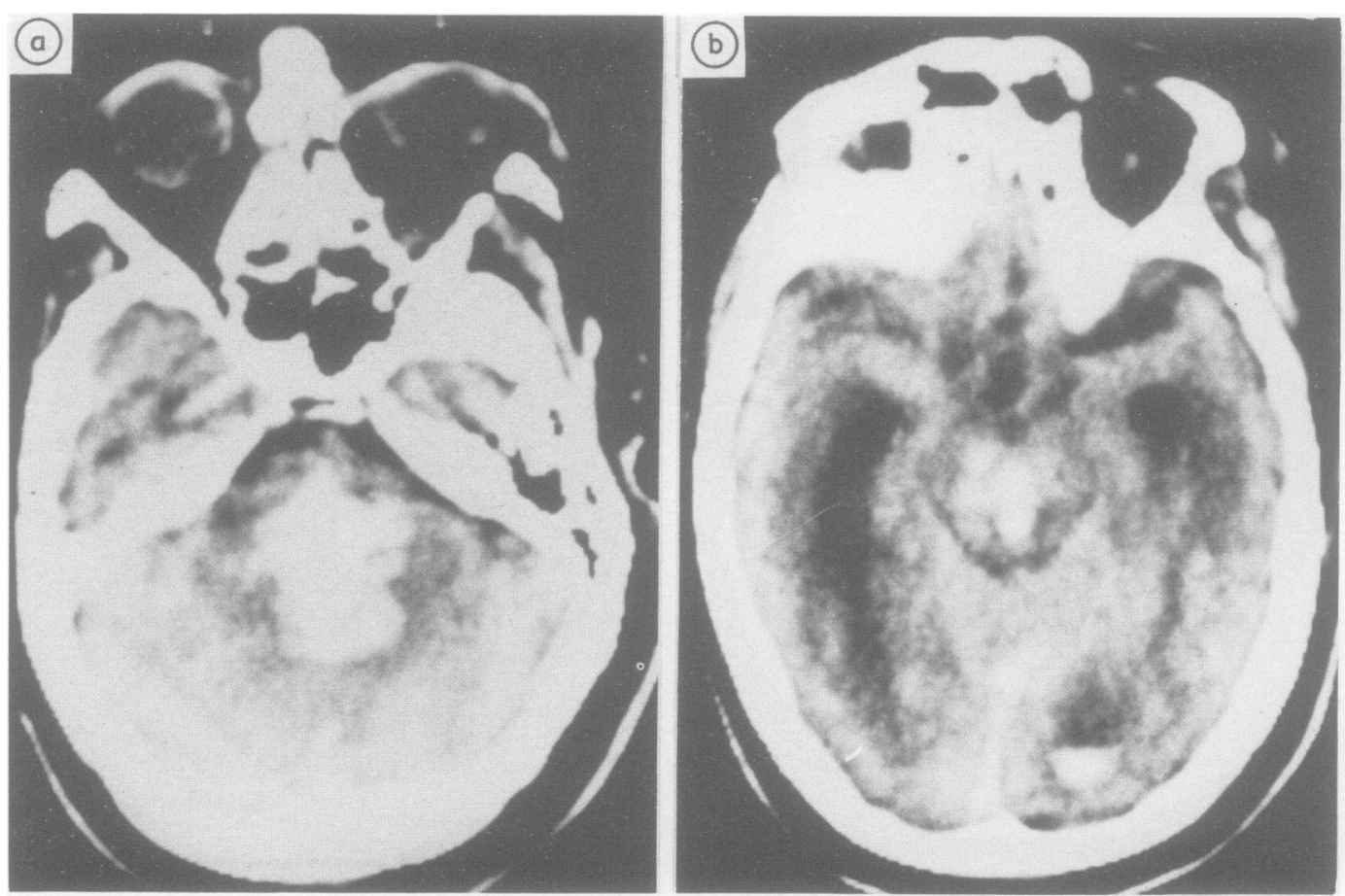

Fig 4 Sixty-eight-year-old normotensive man developed chest pain and collapsed. He was comatose with irregular respiration and quadriplegia. CT findings: large primary pontine haematoma $(a)$ which extends to the midbrain (b).

subarachnoid blood was not seen in any of the other patients with primary pontine haemorrhage. In young and normotensive patients with CT evidence of brain stem haemorrhage, angiography is indicated to delin-

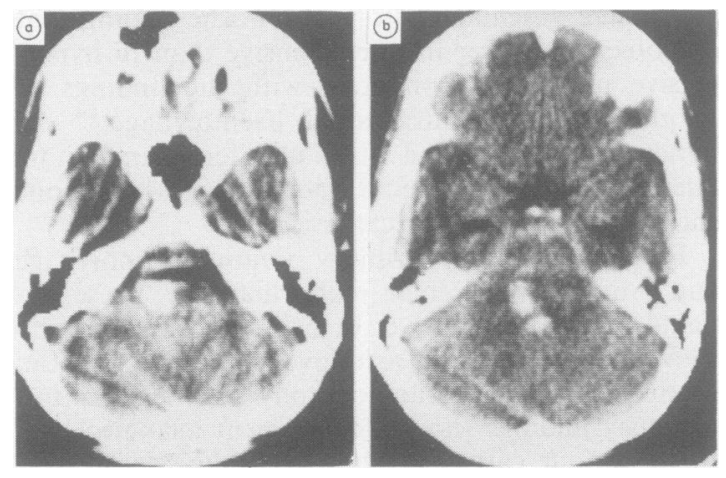

Fig 5 Twenty-nine-year-old normotensive woman developed headache and collapsed. She was comatose with right hemiplegia and absent caloric response. CT findings: right lateral tegmental haematoma $(a)$ with blood also seen in the interpeduncular and ambient cisterns $(b)$. Angiogram showed a brain stem vascular malformation. eate the presence of a brain stem malformation. 121315

It is important to recognise the CT pattern of blood in the fourth ventricle without blood in the brain stem region. Fourth ventricular blood without pontine haematoma is suggestive of ruptured vascular malformation or aneurysm. If blood is seen in the fourth ventricles and brain stem cisterns, this is highly suggestive of ruptured vascular malformation or aneurysm as this pattern was not seen in any cases of hypertensive primary pontine haemorrhage even when there was secondary intraventricular extension. In these cases of brain stem haematoma with evidence of subarachnoid blood, angiography is necessary to determine the source of the haemorrhage. In four cases of primary thalamic haematoma there was a small thalamic hyperdense lesion contiguous with the third ventricle which was seen on the initial CT scan. If the thalamic haematoma is small it may be difficult to differentiate from the third ventricular blood. In these cases blood may be seen extending into the third and fourth ventricles but without blood in the pons (fig 6). Based upon the clinical features and careful CT analysis, it should be possible to differentiate these thalamic haemorrhages from primary pontine haemorrhage or primary intraventricular haemorrhage. 

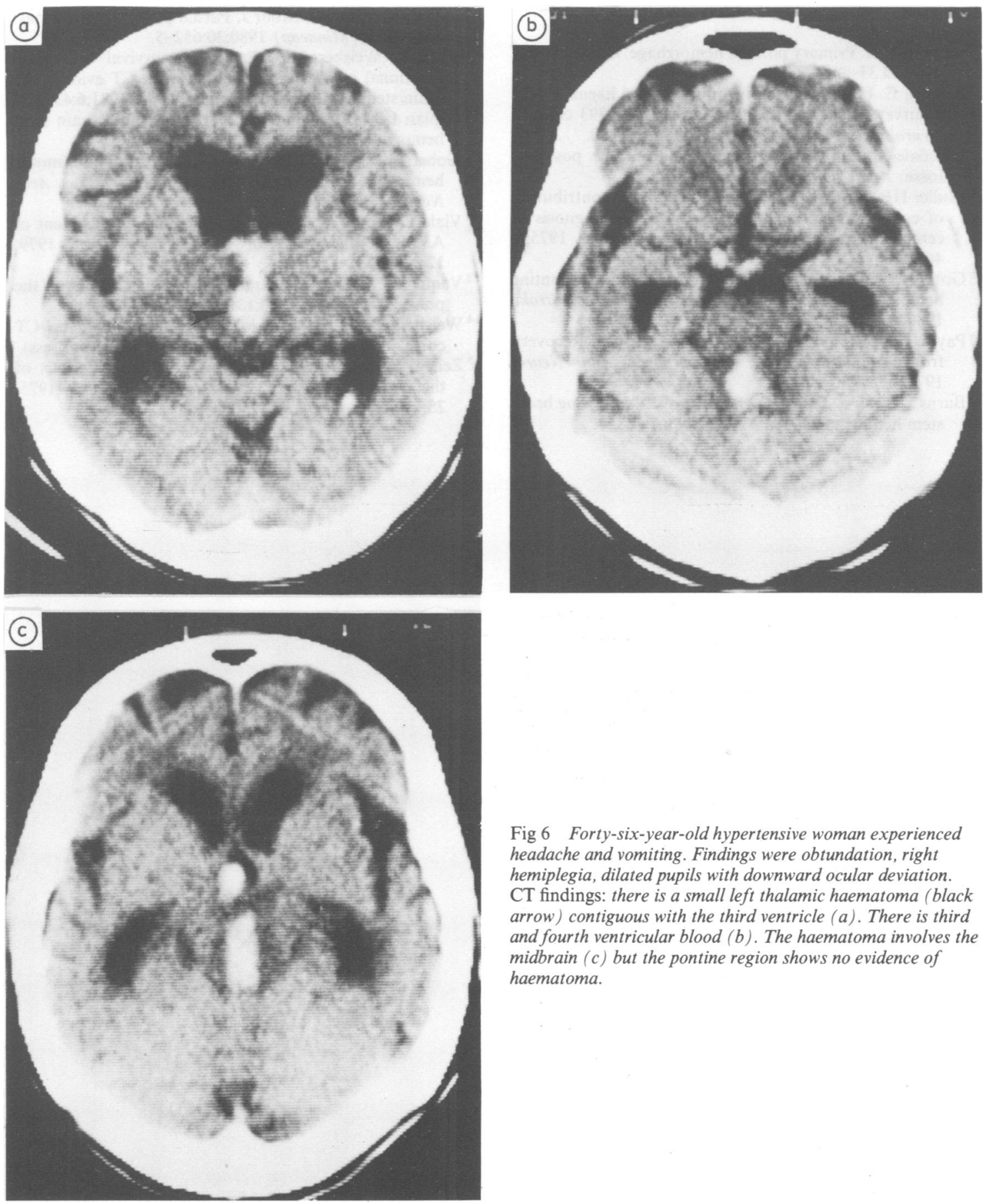

Fig 6 Forty-six-year-old hypertensive woman experienced headache and vomiting. Findings were obtundation, right hemiplegia, dilated pupils with downward ocular deviation. CT findings: there is a small left thalamic haematoma (black arrow) contiguous with the third ventricle (a). There is third and fourth ventricular blood $(b)$. The haematoma involves the midbrain (c) but the pontine region shows no evidence of haematoma. 


\section{References}

${ }^{1}$ Silverstein A. Primary pontine hemorrhage. Confin Neurol 1967;29:33-46.

${ }^{2}$ Freytag E. Fatal hypertensive intracerebral haematomas: a survey of the pathological anatomy of 393 cases. $J$ Neurol Neurosurg Psychiatry 1968;31:616-20.

${ }^{3}$ Dinsdale H. Spontaneous hemorrhage in the posterior fossa. Arch Neurol 1964;10:200-17.

${ }^{4}$ Muller HR, Wuthrich R, Wiggli U, et al. The contribution of computerized and tomography to the diagnosis of cerebellar and pontine hematomas. Stroke 1975;6: 467-75.

${ }^{5}$ Goto N, Kaneko M, Hosaka Y, et al. Primary pontine hemorrhage: clinico-pathological correlations. Stroke 1980;84:90.

${ }^{6}$ Payne HA, Maravilla KR, Levinstone A, et al. Recovery from primary pontine hemorrhage. Ann Neurol 1978;4:557-8.

${ }^{7}$ Burns J, Lisak R, Schert L, et al. Recovery following brain stem hemorrhage. Ann Neurol 1980;7:183-4.
${ }^{8}$ Kase C, Maulsby G, Mohr J. Partial pontine hematomas. Neurology (Minneap) 1980;30:652-5.

${ }^{9}$ Bryan R, Weisberg LA. Prolonged survival with good functional recovery in 3 patients with CT evidence of brain stem hemorrhage. Comput Radiol 1981;6:43-8.

${ }^{10}$ Caplan LR, Goodwin JA. Lateral tegmental brain stem hemorrhages. Neurology (NY) 1982;32:252-60.

${ }^{11}$ Gobernado J, deMolina A, Gimeno A. Pure motor hemiplegia due to hemorrhage in the lower pons. Arch Neurol 1980;37:393-8.

12 Viale GL, Pau A, Schrbundt VE. Surgical treatment of AVM of the posterior fossa. Surg Neurol 1979; 12:379-84.

${ }^{13}$ Vaquero J, Areitio E, Leunda G, et al. Hematomas of the pons. Surg Neurol 1980;13:115-18.

14 Weisberg LA. Subcortical lobar haematomas: clinical-CT correlations. J Neurol Neurosurg Psychiatry (in press).

${ }^{15}$ Zeller RS, Chutorian AM. Vascular malformations of the pons in children. Neurology (Minneap) 1975; 25:776-80. 